\title{
Bioengineering Strategies to Treat Female Infertility
}

\author{
Che-Ying Kuo, MS,1,2 Hannah Baker, PhD, ${ }^{1}$ Melissa H. Fries, MD, James J. Yoo, MD, \\ Peter C.W. Kim, MD, PhD, 2,5 and John P. Fisher, $\mathrm{PhD}^{1,2}$
}

Bioengineering strategies have demonstrated enormous potential to treat female infertility as a result of chemotherapy, uterine injuries, fallopian tube occlusion, massive intrauterine adhesions, congenital uterine malformations, and hysterectomy. These strategies can be classified into two broad categories as follows: (i) Transplantation of fresh or cryopreserved organs into the host and (ii) tissue engineering approaches that utilize a combination of cells, growth factors, and biomaterials that leverages the body's inherent ability to regenerate/ repair reproductive organs. While whole organ transplant has demonstrated success, the source of the organ and the immunogenic effects of allografts remain challenging. Even though tissue engineering strategies can avoid these issues, their feasibilities of creating whole organ constructs are yet to be demonstrated. In this article we summarize the recent advancements in the applications of bioengineering to treat female infertility.

Keywords: biomaterial, bioengineering, tissue engineering, uterus, ovary, follicle culture, cervicovaginal, female infertility

\section{Introduction}

A CCORDING TO THE Centers for Disease Control and Prevention, $10.9 \%$ of women (6.7 million) in the United States ages 15-44 have impaired fecundity. ${ }^{1,2}$ While infertility therapies such as in vitro fertilization (IVF) have made great strides in treating female infertility, there remain structural aspects of female infertility that have not been addressed. Female infertility has endocrine, structural, and iatrogenic causes, including chemotherapy, uterine injuries, fallopian tube occlusion, massive intrauterine adhesions, congenital uterine malformations, and hysterectomy (Fig. 1). ${ }^{3-5}$ Women suffering from congenital and acquired uterine factor infertility are currently treated with approaches such as gestational surrogacy, metroplasty, hysteroscopic repair, and/or hormonal therapies. ${ }^{3-5}$ However, there are issues associated with these approaches. For example, gestational surrogacy is expensive and ethically controversial ${ }^{6,7}$ and surgical approaches may not be applicable to all. Moreover, hormonal therapies often do not provide physiologic concentrations of hormones to treat infertility for conditions such as premature ovarian failure.

Therefore, significant amount of efforts is devoted to develop bioengineering approaches to restore fertility, which can generally be classified into two broad categories as follows: (i) Transplantation of fresh or cryopreserved organs transplanted into the host (Fig. 2); and (ii) tissue engineering approaches that utilize a combination of cells, growth factors, and biomaterials that leverages the body's inherent ability to regenerate/repair reproductive organs (Fig. 3). Tissue engineering often utilizes a variety of different cell types, which include (i) tissue specific stem cells; (ii) mesenchymal stem cells (MSCs); and (iii) pluripotent stem cells such as embryonic stem cells.

Fresh and cryopreserved organ/tissue transplant is often preferred because they retain the integrity of the extracellular matrix (ECM) structure and the complex cellularity that exist in the natural tissue. The source of tissues/organs can either be allogeneic or autologous. Autologous sources are often preferred as they avoid immune rejections and the significant negative effect of immunosuppressive drugs, but autologous organs/tissues' harvest is often difficult to perform. Due to the limited availability of autologous and allogeneic sources, many researchers have turned to decellularized tissues/organs. Some of the most popular decellularized tissue matrices used in female reproductive organ regeneration are porcine small intestinal submucosa (SIS), peritoneum, and amniotic membrane. While decellularization preserves the architecture

${ }^{1}$ Fischell Department of Bioengineering, University of Maryland College Park, College Park, Maryland.

${ }_{3}^{2}$ Sheikh Zayed Institute for Pediatric Surgical Innovation, Children's National Health System, Washington, District of Columbia.

${ }^{3}$ Obstetrics and Gynecology, MedStar Washington Hospital Center, Washington, District of Columbia.

${ }^{4}$ Wake Forest Institute for Regenerative Medicine, Wake Forest University School of Medicine, Winston-Salem, North Carolina.

${ }^{5}$ School of Medicine and Health Sciences, The George Washington University, Washington, District of Columbia. 


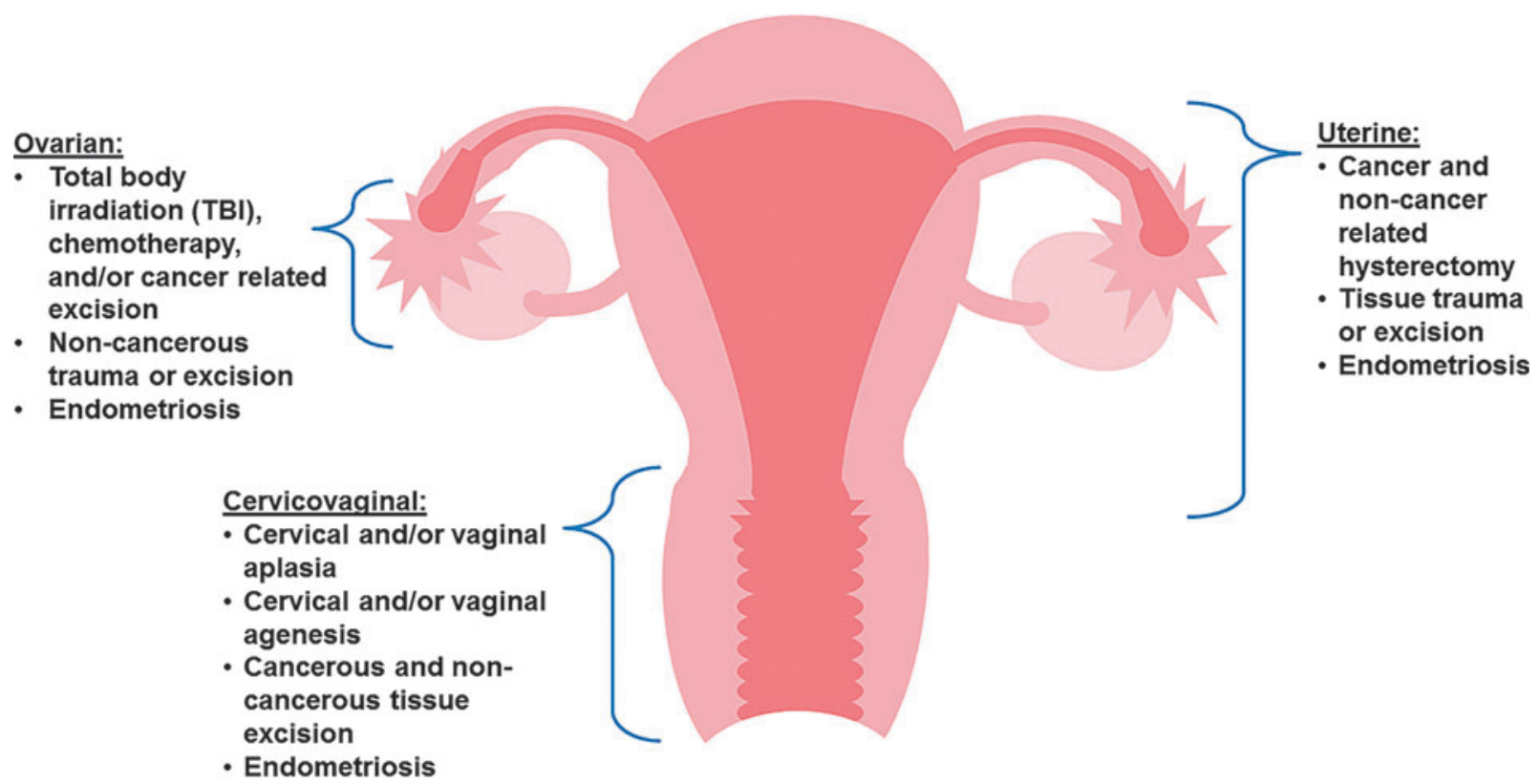

FIG. 1. The female reproductive system and their contributions to female infertility. Cancer and its associated treatments are the major cause of female infertility as they often involve the removal of the entire organ. Other causes of female infertility include traumatic injuries and congenital defects that cause irreversible structural damage.

of the ECM and minimizes the effect of immune rejections, the use of decellularization agents can disrupt the ultrastructure of the decellularized organ, and residual cellular components not removed during processing (e.g., nucleic acid material) can have untoward effects on the host. In addition, preservation of the exact composition of ECM is difficult to characterize, and the residual cellular materials remain a continued concern. Therefore, to allow better control over the composition of biological implants, many researchers have turned to purified natural (e.g., collagen, gelatin, and alginate) or synthetic (e.g., polyethylene-glycol and polypropylene-fumarate) biomaterials.
Many investigators have used natural biomaterials because they inherently support cell adhesion, migration, proliferation, and differentiation. However, the composition of the natural biomaterial must be characterized and there is often significant batch-to-batch variability. Synthetic biomaterials, on the other hand, are well defined and can be fabricated with modifications to molecular structure to enhance biocompatibility. Nevertheless synthetic biomaterials have major disadvantages, including a structure and composition that can only mimic native tissues/organs and a decreased capacity to induce cellular and tissue remodeling.

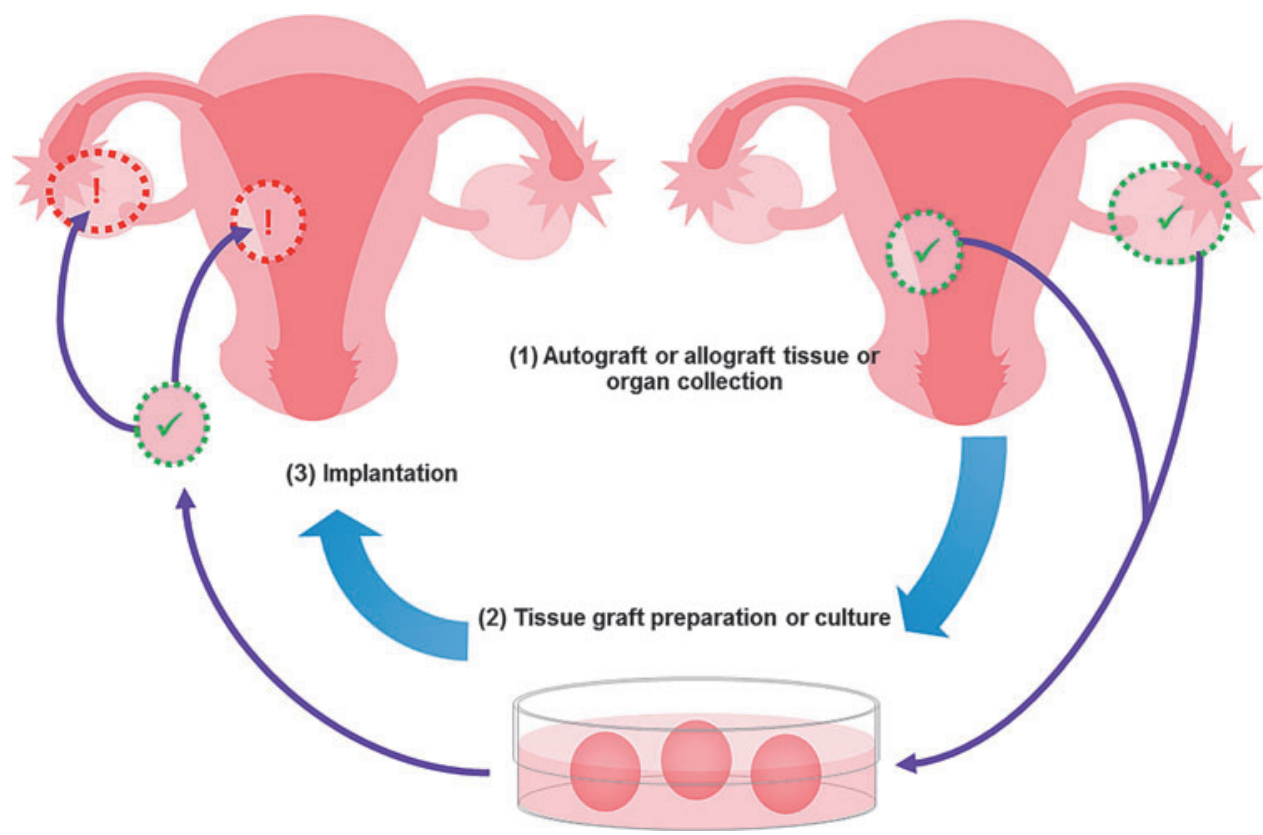

FIG. 2. A schematic of using tissue/organ transplant based on autograft or allograft. First, healthy donor tissue or whole organs are explanted (1) and prepared, which may include static or dynamic culture conditions to apply additional biochem$\mathrm{ical} /$ mechanical stimulus and/or to recellularize the organ/tissue (2). The processed organ/tissue is then used in repairing, reconstructing, or replacing host tissue or organs through surgical implantation (3). 


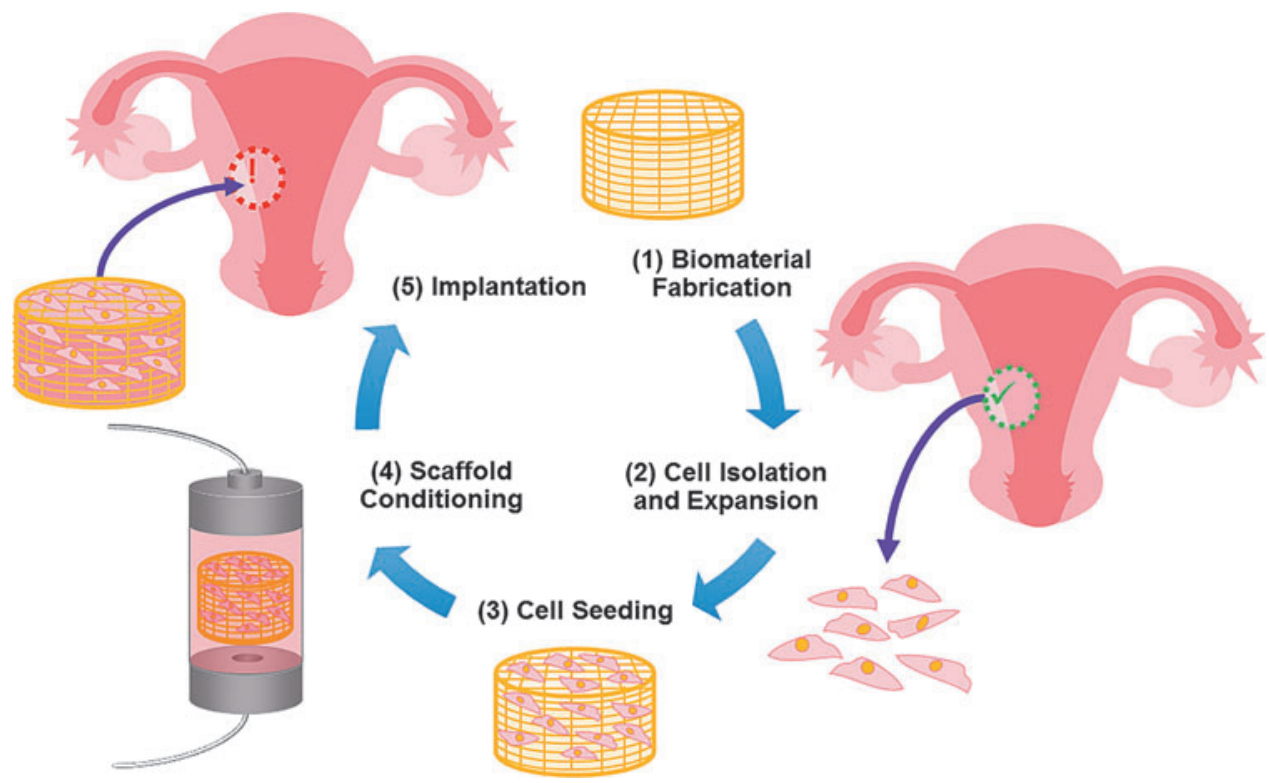

FIG. 3. The fabrication of tissue engineered grafts for female reproductive organ repair involving biomaterials, cells, and/ or biochemical signals. A typical process involves a biomaterial scaffold that is either molded or three-dimensionally printed (1). This scaffold then acts as a carrier that may be loaded with appropriate cell populations (e.g., stem cells or endometrial cells) or bioactive molecules (e.g., epidermal growth factors, vascular endothelial growth factors) (2, 3). The scaffolding loaded with appropriate cells and biochemical signals is then subject to further preconditioning in a bioreactor to apply additional mechanical/biochemical signals, if needed, (4) which is then surgically implanted (5).

In addition, cell source remains a critical challenge in regenerating female reproductive organs. Specifically, a tissue biopsy may not yield sufficient number of normal cells for expansion and transplantation. In other instances, the cells of interest may not be expanded in vitro. Therefore, other investigators explored the possibility of using stem cells as an alternative cell source. Stem cells, with the majority of the work done in syngeneic models, can be obtained from discarded human embryos (human embryonic stem cells [hESCs]), fetal tissue, adult sources (bone marrow, fat, and skin), or may be molecularly induced from differentiated cells (induced pluripotent stem cells). The goal of this review is to present an overview and the most recent advancements in the applications of bioengineering to reestablish female fertility by structural restoration and the associated functions.

\section{Uterus}

Uterine factor infertility, which affects 3-5\% of the general population, can arise from congenital abnormalities or acquired causes such as hysterectomy due to malignant or benign tumor or intrauterine adhesions. ${ }^{3,4,8}$ Adoption and/or gestational surrogacy are currently the only options for having children in these patients. ${ }^{8}$ Current bioengineering approaches for uterine tissue restoration can be broadly classified into uterine transplant and tissue engineered constructs for partial uterine regeneration, which are summarized in Table 1.

\section{Uterus tissue transplant}

The first human trial of whole uterus transplant was performed in 2000 on a female (age 26) who, after a postpartum hemorrhage, lost her uterus 6 years earlier. 9 The donor was a 46-year-old patient with multiloculated ovarian cysts who underwent a hysterectomy modified to preserve tissue and vascular integrity. ${ }^{9}$ The transplanted uterus responded well to combined estrogen-progesterone therapy, but developed acute vascular thrombosis 99 days after transplantation and hysterectomy became necessary. ${ }^{9}$ Therefore, it was evident that further preclinical studies are necessary to demonstrate the establishment and maintenance of revascularization, as well as successful pregnancy of the transplanted uterus.

Since achieving adequate blood supply for the transplanted uterus is essential to clinical success, there have been significant amount of efforts invested in developing different surgical techniques to improve perfusion and viability of uterine autografts. ${ }^{10-13}$ Although these studies only used an autotransplant model and did not test the function/fertility of the uterus, the authors demonstrated surgical techniques and procedures that resulted in successfully perfused uterine tissue with decent viability. In addition to demonstrating uterine perfusion, other studies have shown successful implantation and pregnancy in autotransplanted uterus and that the offspring are healthy and fertile. ${ }^{14-16}$ Based on these results surrounding autotransplantation, another research group moved forward with an allogenic uterus transplantation study. ${ }^{17}$ After uterine transplantation, with vascular anastomoses unilateral to the internal iliac artery and the external iliac vein, the uterus recipients received two different immunosuppressive treatments as follows: (i) monotherapy with tacrolimus and (ii) standard triple therapy. ${ }^{17}$ All the recipients and the donors survived the surgery with recipients presenting rejection to some extent within the first few weeks following uterine transplant. The authors did not conclude which immunosuppressive treatments had better clinical outcomes or if immunosuppressive drugs caused any uterine damage. ${ }^{17}$

Overall, these findings suggest that allogeneic uterine transplant in primate species is feasible and that fresh uterus 


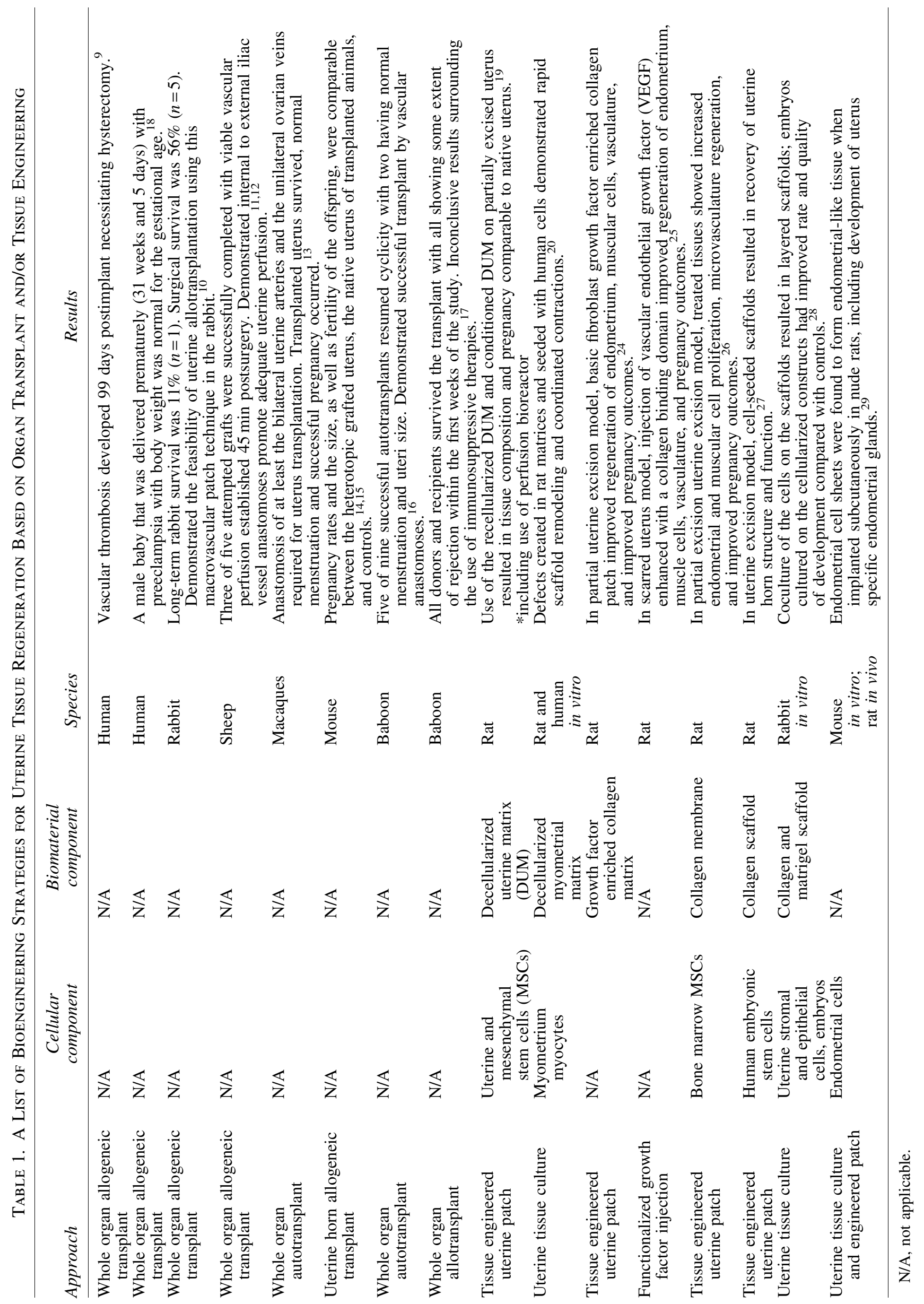


transplant is capable of restoring female fertility. However, there have not been successful term pregnancies in primates to date. Based on these results, there have been 12 human uterus transplantation attempts worldwide but with only one study, performed by Brannstrom et al. in 2013, result in live birth. ${ }^{18}$ In this study, the male baby that was delivered prematurely (31 weeks and 5 days) with preeclampsia with body weight was normal for the gestational age. ${ }^{18}$ Nevertheless this provides a proof-of-concept for the procedure of whole uterine transplant. While issues surrounding immunogenic effects and availability of allogenic tissues remain, uterine transplant is currently the only viable option for whole uterus regeneration. Impact of the medications needed for immunosuppression on the developing fetus will also need continuing evaluation.

\section{Acellular tissue engineering approaches for partial uterus repair}

In an effort to overcome immunogenic and organ availability issues surrounding fresh organ transplant, many investigators have developed tissue engineered constructs for partial uterus repair. Decellularized ECM biomaterials are widely used in these endeavors. ECM based constructs benefit from native tissue protein presence and arrangement, which serve to enhance cell adhesion and migration. For example, Miyazaki and Maruyama aimed to partially regenerate and reconstruct the rat uterus through recellularization of decellularized uterine matrix. ${ }^{19}$ The rat uteri were decellularized by perfusing detergents through the aorta. ${ }^{19}$ Efficient in vitro recellularization was achieved by reseeding of the decellularized uterus, resulting in a reconstitution of an endometrium-like tissue. ${ }^{19}$ This procedure was capable of partially regenerating structurally and functionally competent uterine tissues when placed onto partially excised uterus in rats. ${ }^{19}$

Moreover, Young et al. created a neo-myometrial tissue patch fabricated from culturing isolated myocytes into decellularized rat and human myometrial scaffolds that could be placed over a uterine defect before pregnancy to enhance structural support and reduce the risk of uterine rupture. ${ }^{20}$ The author successfully developed xeno-neo-myometrial grafts that expressed coordinated contractions, which can also serve as a model to study myometrial contractility. ${ }^{20}$ These results are very promising for the use of decellularized ECM for uterine regeneration. However, one of the major disadvantages of using decellularized tissue matrices, as previously mentioned, is the risk of residual cellular materials (e.g., DNA, cells, and antigens), which could trigger significant immune response of the host, which were not considered in these studies. ${ }^{21-23}$

Collagen scaffolds in combination with growth factors have also been investigated as a biomaterial for uterine tissue engineering due to their abundance, biocompatibility, and biodegradability. In one case, a collagen-based basic fibroblast growth factor (bFGF) targeting delivery system was created by fusing a collagen-binding domain (CBD) to the N-terminal of native bFGF (NAT). ${ }^{24}$ Overall, the collagen/CB-bFGF group led to higher endometrium thickness with higher alpha-smooth muscle actin positive areas, more collagen remodeling, neovascularization, and cellularization, ${ }^{24}$ and the collagen scaffold was completely degraded and replaced by endometrial cells and muscle fibers. Em- bryos were found in grafted and normal tissue only in the collagen/CBD-bFGF group and having comparable size to those implanted in the normal tissue. ${ }^{24}$

Using a similar approach, collagen-binding vascular endothelial growth factor (CB-VEGF) was also explored as a potential growth factor for uterine repair, where collagen and CB-VEGF were directly injected into the injury site. ${ }^{25}$ In vivo results showed that the $\mathrm{CB}-\mathrm{VEGF}$ group resulted in a greater thickness of uterine wall regenerated, increased presence of glands, presence of smooth muscle cells, and observed vascular growth. ${ }^{25}$ In terms of fertility, embryos were implanted both in the normal and scar tissue areas of rats in the CBVEGF group, and the pregnancy rate at scar sites in the CBVEGF group was higher than in the NAT-VEGF group and the phosphate buffered saline (PBS) group. ${ }^{25}$ These results are encouraging for restoration of fertility in mammals, but in vivo testing in larger animal models would serve to further minimize the translational distance for these therapies. However, while useful for patch-type applications for partial uterine wall regeneration for implantation, these approaches cannot be used to perform whole uterine regeneration.

\section{Cell-laden tissue engineering approaches for partial uterus repair}

To enhance the regenerative capacity of tissue engineered scaffolds, cell-laden tissue engineered constructs were also explored as a treatment option for uterine regeneration. For instance, collagen membranes seeded with bone marrowderived mesenchymal stem cells (BM-MSCs) were evaluated for their healing capability for severe uterine injury. ${ }^{26}$ Compared with cell-free scaffolds and untreated controls, the collagen/BM-MSC system increased proliferative abilities of uterine endometrial and muscular cells, facilitated microvasculature regeneration, and restored the ability of the endometrium to receive the embryo and support its development to a viable stage. $^{26}$

Endometrium-like cells were generated from the hESCs and were encapsulated within a collagen scaffold to repair uterine damage using rat model of uterine full-thickness injury. $^{27}$ Twelve weeks after transplantation, the authors discovered that the hESC-derived cells could survive and recover the structure and function of uterine horns in a rat model of severe uterine damage. ${ }^{27}$ The pregnancy rate of the cells/collagen group $(80 \%)$ was comparable to that of the sham-operated group $(100 \%)$ and higher compared to the collagen only group $(33 \%)$ and the untreated group $(26.7 \%) .^{27}$ Cell-laden tissue engineered constructs have clearly demonstrated superior regenerative capability over scaffold-only approaches. However, the immunogenic effect and the source of cells need to be considered before clinical translation as autologous cells are typically difficult to obtain.

\section{Cell-based bioengineering approaches for partial uterine repair}

To ensure that the matrix implanted to promote tissue regeneration is biomimetic, some investigators chose to construct a tissue engineered therapy using a cell-only scaffold-free approach. For example, Lu et al. aimed to construct uterine tissue containing a smooth muscle cell layer, an epithelial cell layer, and an endometrial layer. ${ }^{28}$ The engineered uterine tissues (EUTs) were generated by 
first seeding epithelial cells on top of the constructed stromal layer over the smooth muscle layer. ${ }^{28}$ Then the author mixed the rabbit epithelial cell and stromal cells in collagen/ Matrigel to form an endometrial layer and seeded this on top of the rabbit smooth muscle layer. ${ }^{28}$ The constructed EUTs had three layers, including the epithelial layer, stromal layer, and smooth muscle layer, which supported development of fertilized eggs up to blastulas. ${ }^{28}$

Moreover, another research group demonstrated an endometrium tissue engineering approach using a scaffold-free, cell sheet engineering strategy based on primary endometrial cells isolated from immature mice and rats. ${ }^{29}$ After 3 days of culture of the mouse endometrial cells, culture temperature was lowered to induce the detachment of endometrial cells. ${ }^{29}$ After detaching from the dish, an endometrial cell sheet was allowed to shrink significantly by cytoskeletal reorganization, recultured for 3 days to construct a thicker endometrial cell sheet, and then harvested. ${ }^{29}$ Cell sheets were transplanted onto exteriorized muscle using a supporting plate. In vitro results demonstrated that the recultured endometrial cell sheets showed an endometrium-specific hormonal response to estrogen and progesterone, demonstrating that the endometrial cell sheets were found to maintain uterine function in culture. $^{29}$ These cell-only, scaffold-free approaches have shown promise, but their efficacy in supporting the growth of fertilized eggs is yet to be tested against other tissue engineered approaches in animal models.

Overall, bioengineering approaches based on fresh whole uterus transplant have demonstrated success in restoring infertility using animal models. This approach is preferred as the majority of the cells, growth factors, and the ultrastructure is intact, which have proven difficult to replicate. However, most of the studies are performed using syngeneic organ transplant models, which limit clinical translation because autotransplantation of uterus is unlikely to occur in a clinical setting. Allotransplant, on the other hand, is more likely to take place in a clinical setting and this concept has been proven by Brannstrom et al. ${ }^{18}$ where the first live birth after uterus transplantation was demonstrated. However, issues associated with immune rejection, organ source, and ethical issues need to be addressed for allotransplantation for this procedure to be widely used in the clinic. Due to these limitations, many investigators have turned to tissue engineering approaches using cells, growth factors, and biomaterials.

Tissue engineering approaches have advantages over tissue transplant because cell and material sourcing are less of an issue compared to auto- and allograft sourcing. Using animal models, tissue engineering approaches demonstrated partial regeneration of the uterine wall and supported pregnancy and produced fertile offspring. However, current fabrication approaches used in tissue engineering face the difficulties of recreating organ ultrastructure and scaling-up for feasible use in whole uterus regeneration. We expect that these issues will be gradually resolved with the development of more advanced biofabrication approaches such as threedimensional (3D) bioprinting. ${ }^{30}$

\section{Ovary}

Primary ovary deficiency is a leading cause of female infertility that affects one in every 10,000 women before the age of 20 , one in every 1000 women before 30 , and one in
100 women before $400^{31,32}$ Potential causes include radiotherapy and/or chemotherapy, environmental factors, viral infections, metabolic and autoimmune diseases, and genetic alterations. ${ }^{3,432}$ These patients are not able to produce normal levels of hormones or to release oocytes. Current bioengineering approaches to restore ovarian function can be broadly classified as ovarian tissue transplant, tissue engineered ovarian scaffold, and ovarian culture system, as well as cell based therapy (Table 2).

\section{Ovarian follicles and tissue transplant}

Transplantation of cryopreserved ovarian follicles has been a standard clinical practice for decades, with demonstrated clinical success. Likewise, ovarian preservation is presently a vital practice for pediatric cancer patients who have chemotherapy and/or pelvic radiotherapy. This typically involves the transplant of autologous ovaries to areas outside the radiation beam or maintained ex vivo until completion of chemotherapy, when they are returned to the donor. This treatment utilizes autologous ovaries so there are no issues related to rejection or need for immunosuppression. Recently researchers have shown, for the first time, recovered fertility after total body irradiation in addition to chemotherapy. ${ }^{33,34}$ In addition, Silber et al. reported a single series of both fresh and cryopreserved ovarian transplants from one center and demonstrated the success of allogeneic and autologous ovarian transplantation. ${ }^{35}$ Moreover, Laronda et al. demonstrated the feasibility of using decellularized ovary transplants by creating a decellularized ovarian scaffold, recellularized it, and transplanted it to successfully initiate puberty in mice. ${ }^{36}$ However, the authors did not show viable offspring, which is essential for this strategy to be used for human transplants.

The strategies presented in this section based on ovarian tissue transplant have displayed excellent clinical outcome, but they are not suitable for ovarian cancer due to the high risk for reintroducing the tumor from carcinogenic material that may still exist in the ovarian tissue. ${ }^{5,32,36,37}$ Therefore, significant efforts have been invested in developing tissue engineered ovarian tissues and in vitro oocyte culture systems for in vivo implantation.

\section{Tissue engineered ovarian scaffold and ovarian culture system}

To avoid reintroducing the disease, researchers have developed tissue engineered oocyte maturation systems to activate oocytes isolated from cryopreserved ovarian tissues. In 2003, Pangas et al. developed a 3D in vitro culture system based on alginate beads for the growth and the development of individual granulosa cell-oocyte complexes (GOCs). ${ }^{38}$ Ovaries from 12-day-old female mice were dissected. GOCs were collected and encapsulated in alginate beads. Granulosa cells proliferated and oocytes grew in volume in alginate beads with spatial arrangement of GOCs. ${ }^{38}$ The 3D alginate culture system could yield far more oocytes than conventional IVF, as the majority of mammalian oocytes are contained in immature and quiescent follicles. ${ }^{38}$ Moreover, $\mathrm{Xu}$ et al. isolated immature follicles from prepubescent, 16day-old female mice. ${ }^{39}$ Single follicles were pipetted into the middle of each alginate droplet and cross-linked in calcium chloride. ${ }^{39}$ After 8 days of culture, IVF and embryo 


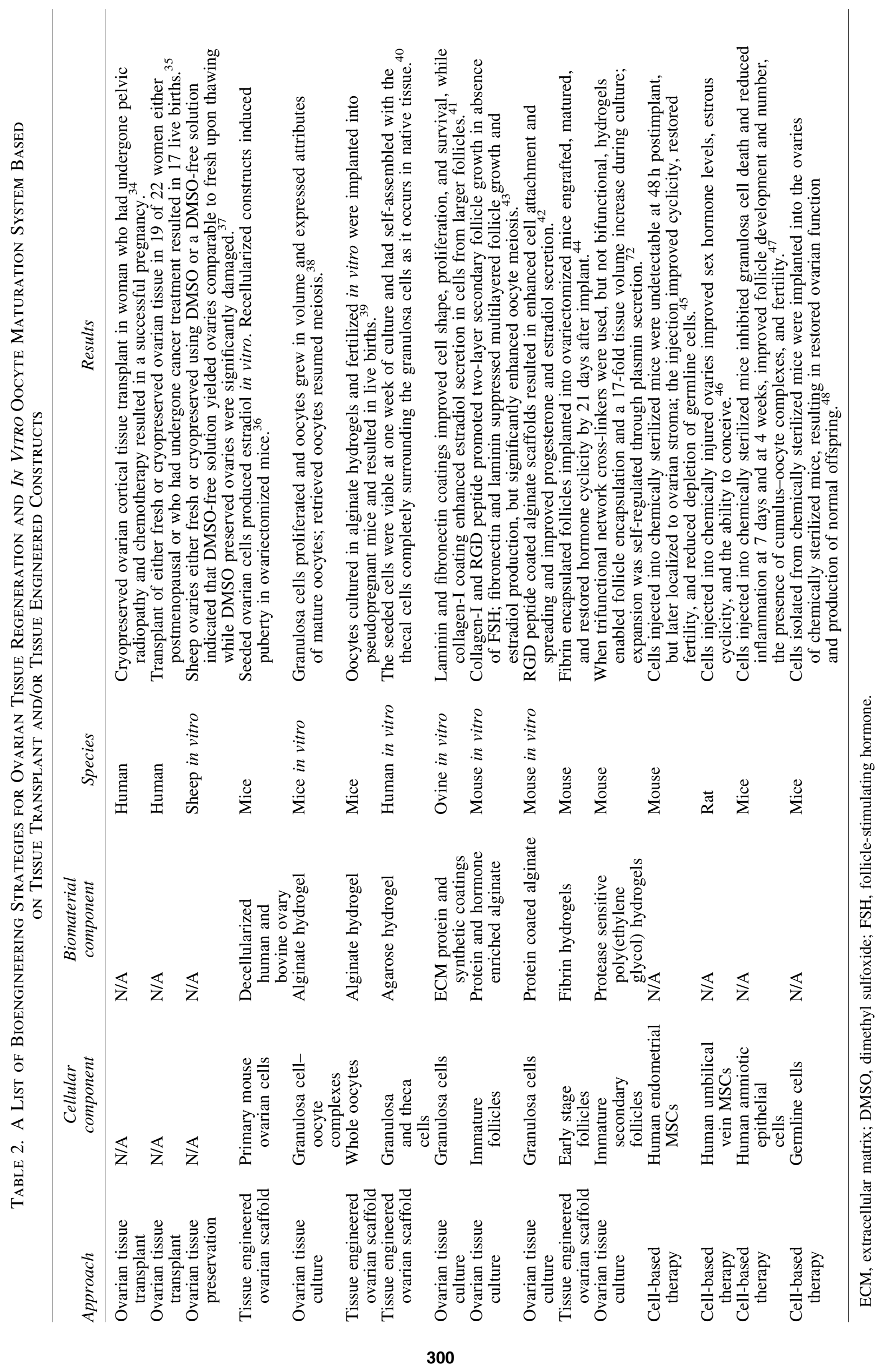




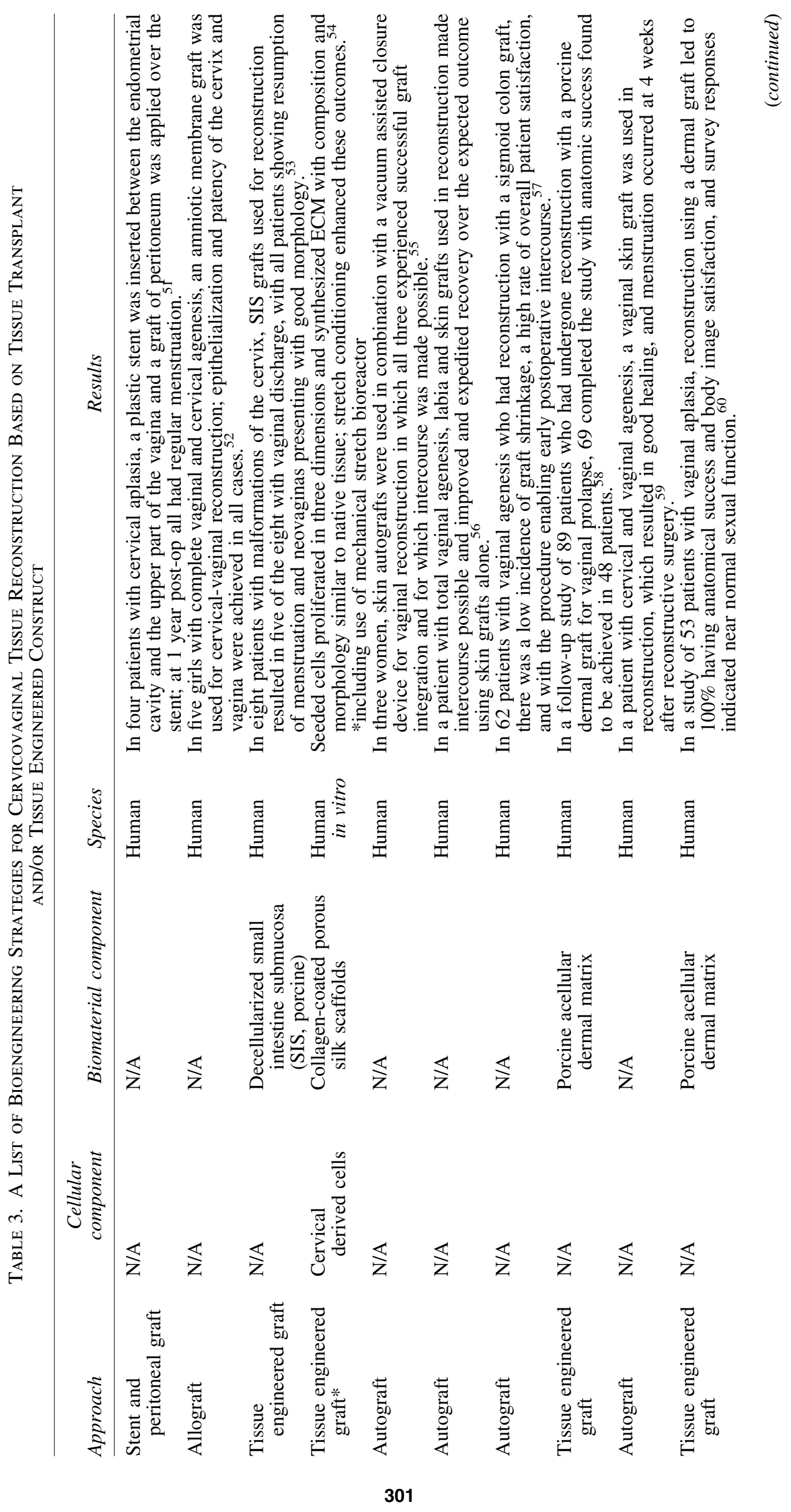




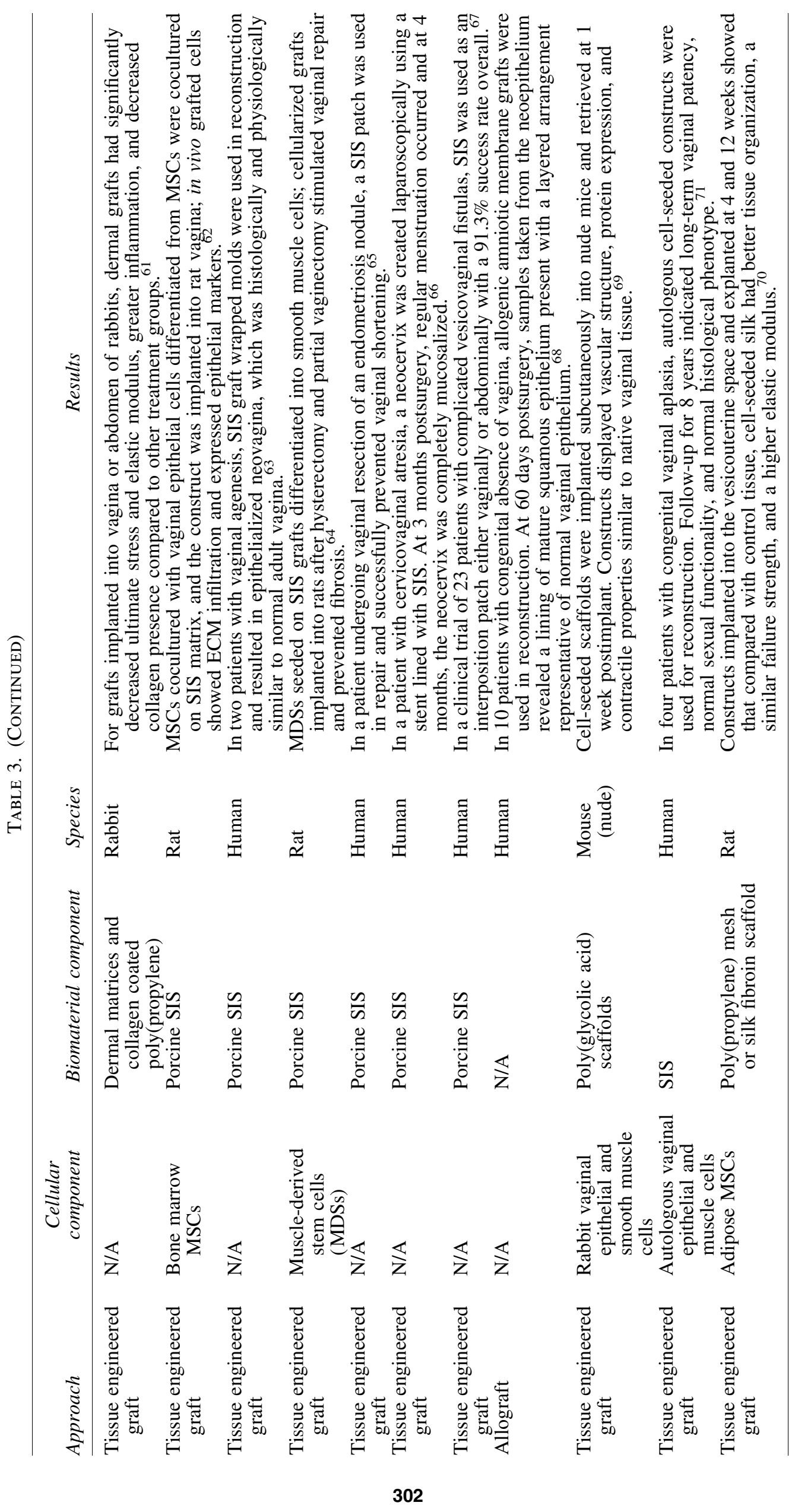


transfer were performed. The embryos derived from cultured oocytes fertilized in vitro and transferred to pseudopregnant female mice were viable, and both male and female offspring were fertile. ${ }^{39}$

The potential of agarose gels, another polysaccharidebased biomaterial that is widely used in tissue engineering, for in vitro maturation of oocytes was investigated. ${ }^{40}$ The authors created a 3D artificial human ovary to mature human oocytes by utilizing the three follicular cell types-theca, granulosa, and oocytes. ${ }^{40}$ The results presenting maturation of early antral follicle $(<10 \mathrm{~mm})$ oocytes into metaphase II oocytes represented the first success in using 3D tissue engineering principles for in vitro oocyte maturation. ${ }^{40}$ However, these approaches using polysaccharide biomaterials alone lack ECM proteins that are essential to the phenotypes of mammalian cells. ${ }^{30}$

As such, other studies utilizing biomaterials which incorporate ECM components such as RGD, collagen (type I and IV), and/or fibronectin into the polysaccharide-based matrix have demonstrated improved growth, differentiation, and meiotic competence of the oocyte. ${ }^{41-43}$ In addition, fibrin was also explored as a potential biomaterial for ovarian tissue engineering. ${ }^{44}$ Fibrin gels with early-stage ovarian follicles encapsulated for transplantation supported their engraftment and, ultimately, development to antral follicles. The fibrin graft was able to reduce systemic follicle-stimulating hormone (FSH) levels and restore cyclicity in a previously noncycling infertile mouse. ${ }^{44}$ These exciting results using hydrogel as model ovarian tissues to provide the necessary cues to activate frozen oocytes created new opportunities for discovery in follicle biology and established a core technology for human egg banks for preservation of fertility. These approaches can potentially develop into a robust system that can be used to consistently activate and mature oocyte. However, issues surrounding natural biomaterials, such as batch-to-batch variability and less-defined molecular composition, need to be resolved before these approaches can be widely utilized clinically.

\section{Cell-based therapy to restore ovarian function}

Many investigators have also explored ovarian tissue engineering approaches without using biomaterials. For example, Lai et al. used human endometrial mesenchymal stem cells (EnSCs) to restore ovarian function through improving the renewal of germline stem cells in a murine model of premature ovarian failure. ${ }^{45}$ Transplanted EnSCs derived from menstrual blood were injected into the tail vein of sterilized mice. EnSC transplantation increased body weight, improved estrous cyclicity, and restored fertility in sterilized mice. ${ }^{45}$ Migration and localization of green fluorescent protein (GFP)labeled EnSCs as measured by live imaging and immunofluorescent methods indicated that GFP-labeled cells were undetectable $48 \mathrm{~h}$ after cell transplantation, but were later detected in and localized to the ovarian stroma. ${ }^{45}$

Moreover, human mesenchymal stem cells isolated from the umbilical cord (HUMSCs) were also tested for their ovarian regeneration capacity. ${ }^{46}$ Membrane-labelled HUMSCs were injected directly into ovary tissue or the tail veins of infertile rats. ${ }^{46}$ HUMSCs injected into the ovary only distributed to the ovary and uterus, while HUMSCs injected through tail vein were detected in the ovary, uterus, kidney, liver, and lung. ${ }^{46}$
The estrous cycle and levels of sex hormones of the treated rats were recovered to a certain degree, and fertility was restored in some transplanted rats with normal development of their offspring. ${ }^{46}$ While ovary injection of HUMSCs could recover ovarian function faster, both delivery methods produced similar results in the later stages of observation, which indicate that transplantation of HUMSCs by tail vein injection represents a minimally invasive and effective treatment method for ovarian injury. ${ }^{46}$

In another study, human amniotic epithelial cells (hAECs) were used to inhibit granulosa cell apoptosis induced by chemotherapy and to restore fertility by injecting cultured hAECs intravenously into infertile mice. ${ }^{47}$ Their results demonstrated that hAEC significantly inhibited cell apoptosis induced by chemotherapeutics, reduced the inflammatory reaction in ovaries, promoted the development of follicles, and increased the number of oocyte complexes in chemoablated mice. ${ }^{47}$ Furthermore, hAECs improved ovarian mass and increased the number of follicles compared to those of the chemoablated group, and hAEC translation partially rescued the fertility of chemoablated mice. ${ }^{47}$ In addition, female germline stem cells (FGSCs) were also shown to rescue ovarian function in chemoablated ovaries. ${ }^{48}$ FGSCs were isolated and purified from adult female mice 2 weeks after chemotherapy. After infection with GFP-carrying virus, the FGSCs were transplanted into ovaries to other genetically identical mice with infertility caused by chemotherapy. Ovarian function was restored and the recipients produced offspring long term. ${ }^{48}$ These findings showed that FGSCs are able to restore ovarian function and avoid immune rejection from exogenous germline stem cells in a chemoablated, infertile mouse model. ${ }^{48}$

Cell-based, scaffold-free approaches to restore ovariancaused infertility are promising based on the results presented in murine models. However, these approaches will need to demonstrate successful pregnancies in larger animal models before clinical translation.

\section{Cervicovaginal Tissue}

Preterm births are a common cause of morbidity and mortality in newborn infants, ${ }^{49}$ and maintenance of the anatomic shape of the cervix during fetal and uterine growth is crucial for a normal pregnancy. ${ }^{3}$ Studies have shown that the relative risk of preterm delivery increases as the length of the cervix decreases, as determined by vaginal ultrasound measurement during pregnancy. ${ }^{50}$

In 2005, Alborzi et al. used a peritoneal graft to treat cervical aplasia. ${ }^{51}$ Through an abdominoperineal approach, a plastic stent was inserted between the endometrial cavity and the upper part of the vagina, and a graft of peritoneum was applied over the stent. ${ }^{51}$ After more than 1 year of observation, all four patients had regular menstrual cycles with normal menstruation with empty uterine cavities, which challenged the standard approach of uterus hysterectomy in case of cervical aplasia recommended by standard gynecological practice. Amniotic membrane and acellular porcine SIS were also demonstrated to be viable options for cervical reconstructions. ${ }^{52,53}$

Then in 2010, House et al. used a tissue engineering approach aimed to develop cervical-like tissue constructs that would be suitable for investigating cervical remodeling. ${ }^{54}$ Specifically, 
cervical cells were isolated from two premenopausal women undergoing hysterectomy for benign gynecological conditions, and the cells were seeded on collagen-coated porous silk scaffolds. Cervical cells proliferated in three dimensions and synthesized an ECM with biochemical constituents and morphology similar to native tissue, and outcomes were enhanced through dynamic culture. ${ }^{54}$ Even though these studies demonstrated the feasibility of constructing cervix-like tissues through bioengineered approaches, which are summarized in Table 3, none of the studies described herein tested the function of cervix during pregnancy and/or under labor conditions. Success in these situations would be critical to translate these technologies to treat female infertility. Nonetheless these strategies have challenged the current standard clinical approach of uterus hysterectomy in cases of cervical aplasia.

Vaginal agenesis, the congenital underdevelopment of the vagina, occurs in 1 out of 5000 females. ${ }^{55-57}$ Various biomaterials have been used to fabricate vaginal grafts, including dermal matrix,${ }^{55,56,58-61}$ SIS, ${ }^{53,62-67}$ and human amniotic membrane. ${ }^{68}$ Recently, there have been several investigators that explored different biomaterials and cell sources to create tissue engineered constructs for vaginal agenesis therapies (Table 3 ). For example, using a rabbit model, vaginal epithelial and smooth muscle cells of female rabbits were grown and expanded in culture and seeded on polyglycolic acid scaffolds. ${ }^{69}$ The cell-seeded scaffolds were subcutaneously implanted into nude mice and the retrieved polymer scaffolds demonstrated multilayered tissue strips of both cell types and penetrating native vasculature noted with contractile properties in response to electrical stimulus. ${ }^{69}$

In addition, adipose-derived MSC seeded silk fibroin scaffolds had a similar failure force and a higher Young's modulus than normal vaginal wall tissue, indicating the potential of this tissue-engineered approach for pelvic floor reconstruction. ${ }^{70} \mathrm{To}$ test the approach clinically, human trials involving autologous epithelial and muscle cells were performed. ${ }^{71}$ Tissue engineered vaginal constructs were then surgically implanted using a perineal approach, and biopsies showed a tri-layered structure, consisting of an epithelial cell-lined lumen surrounded by matrix and muscle, with expected components of vaginal tissue present. The magnetic resonance imagings, which showed the extent of the vaginal aplasia before surgery, showed the engineered organs and the absence of abnormalities after surgery. A validated selfadministered Female Sexual Function Index questionnaire showed variables in the normal range in all areas tested.

The potential of other cell sources was also evaluated by other investigators. For example, skeletal muscle-derived stem cells seeded on SIS were demonstrated to stimulate vaginal repair in rats, indicating a promising approach for vaginal repair. ${ }^{64}$ In another study, BM-MSCs were stimulated to acquire phenotypes of vaginal epithelial cells (VECs) by coculturing with VECs. ${ }^{62}$ BM-MSCs combined with SIS were implanted in the place of the native vagina in rats to observe the potential for vaginal reconstruction. In vivo results demonstrated significant matrix infiltration and expressed epithelial markers in neovagina. ${ }^{62}$ These studies showed that cell-seeded polymer scaffolds were able to form vascularized vaginal tissue in vivo with phenotypic and functional properties similar to those of normal vaginal tissues. However, these studies used autologous cells that were isolated from the host, which can sometimes be lim- ited. Therefore, cell sources need to be identified before we can translate these therapies for use in the operating room. Even so, with the advancement in tissue engineering and the understanding of vaginal biology, we expect to see more tissue engineered strategies being introduced to the clinic.

\section{Conclusion}

Bioengineering approaches have demonstrated enormous potential in treating female infertility, which can be broadly classified into whole organ transplant (Fig. 2) and tissue engineering approaches (Fig. 3). While whole organ transplant has demonstrated success in large animal models, including nonhuman primates, the source of the organ needs to be identified and the immunogenic effects of allografts need to be characterized before the technology becomes a standard clinical practice. Therefore, there has been a growing interest in using tissue engineering approaches to develop substitute tissues/organs as replacements for nonfunctional organs, which would be easier to scale-up for translation. In spite of many investigations into tissue engineered therapies, the feasibility of creating whole organ constructs through these approaches still needs to be demonstrated. Nevertheless, bioengineering strategies demonstrate enormous promise as potential treatments and cures for female infertility, with clinical translation of these technologies undoubtedly on the horizon.

\section{Acknowledgment}

This study was funded by the Sheikh Zayed Institute for Pediatric Surgical Innovation, Children's National Health System.

\section{Disclosure Statement}

No competing financial interests exist.

\section{References}

1. Chandra, A., Martinez, G.M., Mosher, W.D., Abma, J.C., and Jones, J. Fertility, Family Planning, and Reproductive Health of U.S. Women: Data from the 2002 National Survey of Family Growth. Washington, DC: Centers for Disease Control and Prevention, US Department of Health and Human Services, 2005.

2. Chandra, A., Copen, C., and Stephen, E. Infertility and Impaired Fecundity in the United States, 1982-2010: Data from the National Survey of Family Growth. Washington, DC: Centers for Disease Control and Prevention, US Department of Human Services, 2013.

3. Sadri-Ardekani, H., and Atala, A. Regenerative medicine for the treatment of reproductive system disorders: current and potential options. Adv Drug Deliv Rev 82, 145, 2015.

4. Yalcinkaya, T., Sittadjody, S., and Opara, E. Scientific principles of regenerative medicine and their application in the female reproductive system. Maturitas 77, 12, 2014.

5. Atala, A. Tissue engineering of reproductive tissues and organs. Fertil Steril 98, 21, 2012.

6. Ber, R. Ethical issues in gestational surrogacy. Theor Med Bioeth 21, 153, 2000.

7. Duska, L.R., Toth, T.L., and Goodman, A. Fertility options for patients with stages IA2 and IB cervical cancer: presentation of two cases and discussion of technical and ethical issues. Obstet Gynecol 92, 656, 1998. 
8. Arora, K.S., and Blake, V. Uterus transplantation: ethical and regulatory challenges. J Med Ethics 40, 396, 2014.

9. Fageeh, W., Raffa, H., Jabbad, H., and Marzouki, A. Transplantation of the human uterus. Int J Gynecol Obstet 76, 245, 2002.

10. Saso, S., et al. Uterine allotransplantation in a rabbit model using aorto-caval anastomosis: a long-term viability study. Eur J Obstet Gynecol Reprod Biol 182, 185, 2014.

11. Saso, S., et al. Achieving uterine auto-transplantation in a sheep model using iliac vessel anastomosis: a short-term viability study. Acta Obstet Gynecol Scand 94, 245, 2015.

12. Saso, S., Thum, M.Y., Bracewell-Milnes, T., and Smith, J.R. Uterine auto-transplantation in a sheep model using iliac vessel anastomosis. Hum Reprod 30, 449, 2015.

13. Mihara, M., et al. Uterine autotransplantation in cynomolgus macaques: the first case of pregnancy and delivery. Hum Reprod 27, 2332, 2012.

14. El-Akouri, R.R., et al. Heterotopic uterine transplantation by vascular anastomosis in the mouse. $\mathrm{J}$ Endocrinol 174, 10, 2002.

15. El-Akouri, R., Kurlberg, G., and Brannstrom, M. Successful uterine transplantation in the mouse: pregnancy and postnatal development of offspring. Hum Reprod 18, 2018, 2003.

16. Enskog, A., et al. Uterus transplantation in the baboon: methodology and long-term function after auto-transplantation. Hum Reprod 25, 1980, 2010.

17. Johannesson, L., et al. Preclinical report on allogeneic uterus transplantation in non-human primates. Hum Reprod 28, 189, 2013.

18. Brannstrom, M., et al. Live birth after uterus transplantation. Obstet Gynecol Surv 70, 394, 2015.

19. Miyazaki, K., and Maruyama, T. Partial regeneration and reconstruction of the rat uterus through recellularization of a decellularized uterine matrix. Biomaterials 35, 8791, 2014.

20. Young, R., and Goloman, G. Allo- and xeno-reassembly of human and rat myometrium from cells and scaffolds. Tissue Eng Part A 19, 2112, 2013.

21. Konakci, K.Z., et al. Alpha-gal on bioprostheses: xenograft immune response in cardiac surgery. Eur J Clin Invest 35, 17, 2005.

22. Badylak, S.F. Xenogeneic extracellular matrix as a scaffold for tissue reconstruction. Transpl Immunol 12, 367, 2004.

23. Badylak, S., Gilbert, T., and Myers-Irvin, J. The extracellular matrix as a biologic scaffold for tissue engineering. In: Thomsen, P., et al. eds. Tissue Engineering Academic Press Series in Biomedical Engineering. London, UK: Elsevier Academic Press, Inc., 2008, pp. 121-143.

24. Li, X., et al. Regeneration of uterine horns in rats by collagen scaffolds loaded with collagen-binding human basic fibroblast growth factor. Biomaterials 32, 8172, 2011.

25. Lin, N., et al. The effect of collagen-binding vascular endothelial growth factor on the remodeling of scarred rat uterus following full-thickness injury. Biomaterials 33, 1801, 2012.

26. Ding, L.J., et al. Transplantation of bone marrow mesenchymal stem cells on collagen scaffolds for the functional regeneration of injured rat uterus. Biomaterials 35, 4888, 2014.

27. Song, T., et al. Regeneration of uterine horns in rats using collagen scaffolds loaded with human embryonic stem cell-derived endometrium-like cells. Tissue Eng Part A 21, 353, 2015.

28. Lu, S., et al. Reconstruction of engineered uterine tissues containing smooth muscle layer in collagen/matrigel scaffold in vitro. Tissue Eng Part A 15, 1611, 2009.
29. Takagi, S., et al. Reconstruction of functional endometriumlike tissue in vitro and in vivo using cell sheet engineering. Biochem Biophys Res Commun 446, 335, 2014.

30. Kuo, C.-Y., et al. Development of a 3D printed, bioengineered placenta model to evaluate the role of trophoblast migration in preeclampsia. ACS Biomater Sci Eng 2, 1817, 2016.

31. Hewlett, M., and Mahalingaiah, S. Update on primary ovarian insufficiency. Curr Opin Endocrinol Diab Obes 22, 483, 2015.

32. Qin, Y.Y., Jiao, X., Simpson, J.L., and Chen, Z.J. Genetics of primary ovarian insufficiency: new developments and opportunities. Hum Reprod Update 21, 787, 2015.

33. Larsen, E., et al. Oocyte donation in women cured of cancer with bone marrow transplantation including total body irradiation in adolescence. Hum Reprod 15, 1505, 2000.

34. Rodriguez-Wallberg, K., et al. Full-term newborn after repeated ovarian tissue transplants in a patient treated for Ewing sarcoma by sterilizing pelvic irradiation and chemotherapy. Acta Obstet Gynecol Scand 94, 324, 2015.

35. Silber, S., Pineda, J., Lenahan, K., DeRosa, M., and Melnick, J. Fresh and cryopreserved ovary transplantation and resting follicle recruitment. Reprod Biomed Online 30, 643, 2015.

36. Laronda, M.M., et al. Initiation of puberty in mice following decellularized ovary transplant. Biomaterials 50, 20, 2015.

37. Du, T.Q., et al. Successful cryopreservation of whole sheep ovary by using DMSO-free cryoprotectant. J Assist Reprod Genet 32, 1267, 2015.

38. Pangas, S.A., Saudye, H., Shea, L.D., and Woodruff, T.K. Novel approach for the three-dimensional culture of granulosa cell-oocyte complexes. Tissue Eng 9, 1013, 2003.

39. Xu, M., Kreeger, P.K., Shea, L.D., and Woodruff, T.K. Tissue-engineered follicles produce live, fertile offspring. Tissue Eng 12, 2739, 2006.

40. Krotz, S.P., et al. In vitro maturation of oocytes via the prefabricated self-assembled artificial human ovary. J Assist Reprod Genet 27, 743, 2010.

41. Huet, C., Pisselet, C., Mandon-Pepin, B., Monget, P., and Monniaux, D. Extracellular matrix regulates ovine granulosa cell survival, proliferation and steroidogenesis: relationships between cell shape and function. J Endocrinol 169, 347, 2001.

42. Kreeger, P.K., Woodruff, T.K., and Shea, L.D. Murine granulosa cell morphology and function are regulated by a synthetic Arg-Gly-Asp matrix. Mol Cell Endocrinol 205, 1, 2003.

43. Kreeger, P.K., Deck, J.W., Woodruff, T.K., and Shea, L.D. The in vitro regulation of ovarian follicle development using alginate-extracellular matrix gels. Biomaterials 27, 714, 2006.

44. Smith, R.M., et al. Fibrin-mediated delivery of an ovarian follicle pool in a mouse model of infertility. Tissue Eng Part A 20, 3021, 2014.

45. Lai, D.M., et al. Human endometrial mesenchymal stem cells restore ovarian function through improving the renewal of germline stem cells in a mouse model of premature ovarian failure. J Transl Med 13, 155, 2015.

46. Zhu, S.F., et al. Human umbilical cord mesenchymal stem cell transplantation restores damaged ovaries. J Cell Mol Med 19, 2108, 2015.

47. Zhang, Q.W., et al. Human amniotic epithelial cells inhibit granulosa cell apoptosis induced by chemotherapy and restore the fertility. Stem Cell Res Ther 6, 152, 2015. 
48. Xiong, J.Q., et al. Intraovarian transplantation of female germline stem cells rescue ovarian function in chemotherapyinjured ovaries. PLoS One 10, e0139824, 2015.

49. Saigal, S., and Doyle, L.W. Preterm birth 3-an overview of mortality and sequelae of preterm birth from infancy to adulthood. Lancet 371, 261, 2008.

50. Iams, J.D., et al. The length of the cervix and the risk of spontaneous premature delivery. N Engl J Med 334, 567, 1996.

51. Alborzi, S., Momtahan, M., Parsanezhad, M.E., and Yazdani, M. Successful treatment of cervical aplasia using a peritoneal graft. Int J Gynecol Obstet 88, 299, 2005.

52. Mhaskar, R. Amniotic membrane for cervical reconstruction. Int J Gynecol Obstet 90, 123, 2005.

53. Ding, J.X., Chen, X.J., Zhang, X.Y., Zhang, Y., and Hua, K.Q. Acellular porcine small intestinal submucosa graft for cervicovaginal reconstruction in eight patients with malformation of the uterine cervix. Hum Reprod 29, 677, 2014.

54. House, M., Sanchez, C.C., Rice, W.L., Socrate, S., and Kaplan, D.L. Cervical tissue engineering using silk scaffolds and human cervical cells. Tissue Eng Part A 16, 2101, 2010.

55. Hallberg, H., and Holmstrom, H. Vaginal construction with skin grafts and vacuum-assisted closure. Scand J Plast Reconstr Surg Hand Surg 37, 97, 2003.

56. Baytekin, C., et al. Total vaginal reconstruction with combined 'Split Labia Minora Flaps' and full-thickness skin grafts. J Obstet Gynaecol Res 33, 524, 2007.

57. Imparato, E., Alfei, A., Aspesi, G., Meus, A.L., and Spinillo, A. Long-term results of sigmoid vaginoplasty in a consecutive series of 62 patients. Int Urogynecol J 18, 1465, 2007.

58. Mahdy, A., Karp, D., Davila, G.W., and Ghoniem, G.M. The outcome of transobturator anterior vaginal wall prolapse repair using porcine dermis graft: intermediate term follow-up. Int Braz J Urol 39, 506, 2013.

59. Lee, C.L., et al. Laparoscopically assisted full thickness skin graft for reconstruction in congenital agenesis of vagina and uterine cervix. Hum Reprod 14, 928, 1999.

60. Zhu, L., Zhou, H.M., Sun, Z.X., Lou, W.J., and Lang, J.H. Anatomic and sexual outcomes after vaginoplasty using tissue-engineered biomaterial graft in patients with MayerRokitansky-Kuster-Hauser syndrome: a new minimally invasive and effective surgery. J Sex Med 10, 1652, 2013.

61. Hilger, W.S., et al. Histological and biomechanical evaluation of implanted graft materials in a rabbit vaginal and abdominal model. Am J Obstet Gynecol 195, 1826, 2006.

62. Li, Y.A., et al. Bone marrow mesenchymal stem cells could acquire the phenotypes of epithelial cells and accelerate vaginal reconstruction combined with small intestinal submucosa. Cell Biol Int 39, 1225, 2015.
63. Ding, J.X., Zhang, X.Y., Chen, L.M., and Hua, K.Q. Vaginoplasty using acellular porcine small intestinal submucosa graft in two patients with Meyer-von-RokitanskyKuster-Hauser syndrome: a prospective new technique for vaginal reconstruction. Gynecol Obstet Invest 75, 93, 2013.

64. Ho, M.H., et al. Stimulating vaginal repair in rats through skeletal muscle-derived stem cells seeded on small intestinal submucosal scaffolds. Obstet Gynecol 114, 300, 2009.

65. Lemos, N., et al. Small intestinal submucosa patch for extensive vaginal endometriosis resection. J Minim Invasive Gynecol 16, 765, 2009.

66. Zhou, Q.J., et al. Laparoscopic-assisted uterovaginal anastomosis for uterine cervix atresia with vaginal aplasia using a silicone stent lined with acellular porcine small intestinal submucosa graft inserted using a $16 \mathrm{~F}$ foley catheter. J Minim Invasive Gynecol 20, 710, 2013.

67. Farahat, Y.A., et al. Application of small intestinal submucosa graft for repair of complicated vesicovaginal fistula: a pilot study. J Urol 188, 861, 2012.

68. BleggiTorres, L.F., Werner, B., and Piazza, M.J. Ultrastructural study of the neovagina following the utilization of human amniotic membrane for treatment of congenital absence of the vagina. Braz J Med Biol Res 30, 861, 1997.

69. de Filippo, R.E., Yoo, J.J., and Atala, A. Engineering of vaginal tissue in vivo. Tissue Eng 9, 301, 2003.

70. Li, Q., Wang, J.L., Liu, H.F., Xie, B., and Wei, L.H. Tissue-engineered mesh for pelvic floor reconstruction fabricated from silk fibroin scaffold with adipose-derived mesenchymal stem cells. Cell Tissue Res 354, 471, 2013.

71. Raya-Rivera, A.M., et al. Tissue-engineered autologous vaginal organs in patients: a pilot cohort study. Lancet 384, 329, 2014.

72. Shikanov, A., Smith, R.M., Xu, M., Woodruff, T.K., and Shea, L.D. Hydrogel network design using multifunctional macromers to coordinate tissue maturation in ovarian follicle culture. Biomaterials 32, 2524, 2011.

Address correspondence to: John P. Fisher, PhD Fischell Department of Bioengineering University of Maryland College Park $2330 D$ Jeong H. Kim Engineering Building 8228 Paint Branch Drive College Park, MD 20742

E-mail: jpfisher@umd.edu

Received: September 21, 2016

Accepted: November 22, 2016

Online Publication Date: February 3, 2017 